\title{
Influence of Semiconductor Bands Bending on Exciton Photoluminescence
}

\author{
A. KONIN* \\ Semiconductor Physics Institute \\ Goštauto 11, LT-01108 Vilnius, Lithuania
}

\begin{abstract}
A theoretical model for calculation of the Wannier-Mott exciton distribution in semiconductor sample accounting for the energy bands bending near semiconductor surface is presented. It is shown that the exciton distribution essentially depends on the surface potential under certain sample surface and bulk parameters. Changing the surface potential value we can study the exciton photoluminescence from the illuminated surface and from a thin layer near this surface.
\end{abstract}

PACS numbers: 71.35.--y, 71.35.Cc

\section{Introduction}

Electro-optic interactions involving excitons in semiconductor microstructures and microdevices are currently attracting much interest [1]. Whereas the localisation of charged particles, like electrons or holes, is easily achievable in electrostatic potential [2], the localisation of neutral excitons needs more special mechanism. The spatial localisation of excitons has been realised in semiconductors by various approaches [3-5]. It is possible to change exciton localisation by mechanical stress through band gap. It is known that a bending of semiconductor energy bands creates a built-in equilibrium electric field $E_{\text {eq }}[6]$ near a sample surface, which can be changed in wide ranges by the known techniques [7]. The inhomogeneous built-in electric field polarises the excitons and therefore, can localise them near the illuminated surface.

This article investigates the exciton photoluminescence (PL) from the illuminated surface and from the nearly bulk due to the exciton redistribution.

\section{Theory}

Let us consider the $n$-type semiconductor plate in the region $0 \leq x \leq L$ with the surface coordinate at $x=0$ which is illuminated by strongly absorbed light. The thickness $L$ essentially exceeds the diffusion length of electron-hole pair (see

*e-mail: konin@pfi.lt 
below). A semitransparent metallic contact is placed on the transparent insulating layer, which is placed on surface $x=0$ of the sample (Fig. 1). The ohmic metallic contact is placed on the opposite surface at $x=L$. The non-equilibrium excitons arise in a sample as a result of electron-hole pair (EHP) recombination.

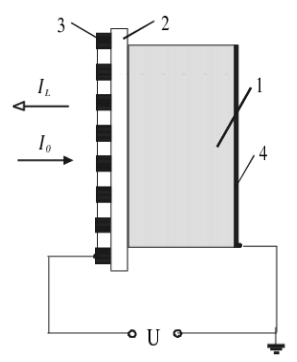

Fig. 1. Experimental setup for measurement of the exciton PL dependence on the energy bands bending. 1 is the semiconductor sample, 2 is transparent insulating plate, 3 is the semitransparent metallic contact, 4 is the grounded metallic contact and $U$ is the external voltage source.

The photoluminescence intensity $I_{L}\left(\lambda_{\mathrm{ex}}\right)$ at the light wavelength $\lambda_{\mathrm{ex}}$, corresponding to the exciton recombination energy, depends on the exciton density distribution by relation

$$
I_{L}\left(\lambda_{\text {ex }}\right) \propto\left[\int_{0}^{L} \frac{n(x)}{\tau} \exp (-\alpha x) \mathrm{d} x+S n(0)\right],
$$

where $n(x)$ is the exciton density, $\tau$ is the exciton lifetime, $S$ is the exciton surface recombination rate (SRR), and $\alpha=\alpha\left(\lambda_{\text {ex }}\right)$ is the light absorption coefficient.

The Wannier-Mott exciton density $n$ is obtained from solution of the continuity equation

$$
\frac{\mathrm{d} j}{\mathrm{~d} x}+\frac{n}{\tau}-G \exp \left(-\frac{x}{\lambda^{*}}\right)=0, \quad j=2 n \mu R \frac{\mathrm{d}\left|E_{\text {eq }}\right|}{\mathrm{d} x}-D \frac{\mathrm{d} n}{\mathrm{~d} x},
$$

and the boundary condition (BC) $j(0)=-S n(0)$. Here $j, \mu, D$ are the exciton flux, mobility, and diffusion coefficient consequently, $R$ is the exciton radius, $G$ is the exciton bulk generation rate at $x=0$, and $\lambda^{*}$ is the EHP diffusion length.

The case when both the EHP diffusion length and the exciton diffusion length essentially exceed the Debye length is analysed. Under these conditions the solution of Eq. (2.2) could be obtained as a sum of the diffusion-recombination (DR) mode and the screening (S) mode [2]:

$$
\begin{aligned}
& n(x)=n_{\mathrm{R}}(x)+n_{\mathrm{S}}(x), \\
& n_{\mathrm{R}}=\frac{G \tau \lambda^{* 2}}{\left(\lambda^{* 2}-\lambda^{2}\right)}\left[\exp \left(-\frac{x}{\lambda^{*}}\right)-\frac{\tilde{\nu} \lambda^{*}+\lambda}{(\tilde{\nu}+1) \lambda^{*}} \exp \left(-\frac{x}{\lambda}\right)\right],
\end{aligned}
$$




$$
n_{\mathrm{S}}=\frac{G \tau \lambda^{*}}{\left(\lambda^{*}+\lambda\right)(1+\tilde{\nu})}\left[\exp \left(\frac{2 \mu R}{D}\left|E_{\mathrm{eq}}\right|\right)-1\right],
$$

where $\lambda=\sqrt{D \tau}$ is the EHP diffusion length and $\tilde{\nu}=(S \tau / \lambda) \exp \left(2 \mu R\left|E_{\text {eq }}(0)\right| / D\right)$ is the effective normalised exciton SRR.

\section{Discussion}

It follows from the $\tilde{\nu}$ expression that the effective normalised exciton SRR strongly increases when the built-in electric field on the illuminated surface fulfils the condition

$$
\left|E_{\text {eq }}(0)\right|>(2 \div 3) D / 2 R \mu \text {. }
$$

The increase in the surface potential $(\mathrm{SP}) \varphi^{\mathrm{S}}$ modulus results in increase in both the built-in electric field modulus and the effective normalised exciton SRR value $\tilde{\nu}$. The density of the $\mathrm{S}$ mode increases under condition $S \ll \lambda / \tau$ and the density of the DR mode decreases. Thus, the excitons are accumulated near the illuminated surface.
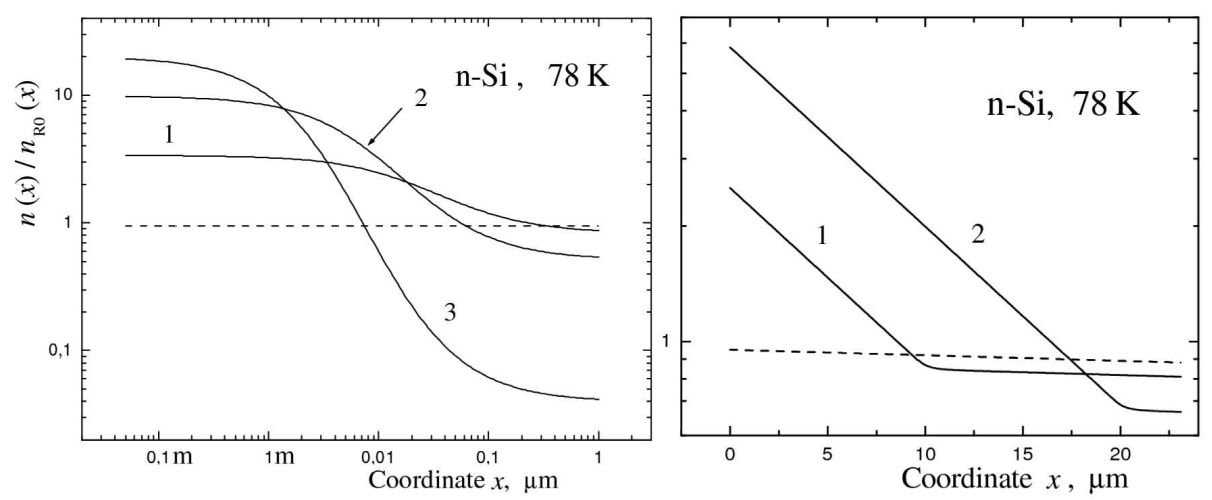

Fig. 2. Exciton density distribution in pure $n$-Si for some surface potential $\varphi^{\mathrm{S}}$ values: (left part) positive: $1-$ for $\varphi^{\mathrm{S}}=40 \mathrm{mV}, 2-$ for $\varphi^{\mathrm{S}}=50 \mathrm{mV}, 3-$ for $\varphi^{\mathrm{S}}=60 \mathrm{mV}$; (right part) negative: $1-$ for $\varphi^{\mathrm{S}}=-100 \mathrm{mV}, 2-$ for $\varphi^{\mathrm{S}}=-200 \mathrm{mV}$. The dashed line gives the $n(x) / n_{\mathrm{R} 0}(x)$ value for flat energy bands.

The normalised exciton density $n(x) / n_{\mathrm{R} 0}(x)$ distribution near the surface $x=0$ in pure $\operatorname{Si}\left(T=78 \mathrm{~K}, n_{0}=2 \times 10^{13} \mathrm{~cm}^{-3}, R_{\mathrm{D}}=0.012 \mu \mathrm{m}, r_{\mathrm{D}}=0.48 \mu \mathrm{m}\right.$, $S \tau / \lambda=0.05)$ is shown in Fig. 2. $r_{\mathrm{D}}$ is the Debye length and $n_{\mathrm{R} 0}(x)$ is the exciton density for flat energy bands and negligible SRR value. It follows from Fig. 2 that the exciton density distribution depends strongly on the SP value. Thus, changing the surface potential value we can change essentially the exciton density distribution and study the exciton PL caused by the surface recombination and by the bulk recombination from a thin layer near the illuminated surface.

In conclusion, the Wannier-Mott exciton distribution accounting for the energy bands bending near semiconductor surface has been presented. It is shown 
that the exciton density distribution near the illuminated surface and in the bulk of the sample essentially depends on the surface potential for small enough SRR. Changing the surface potential value we can study the exciton PL from the illuminated surface and from a thin layer near this surface.

\section{References}

[1] F. Henneberger, S. Schmitt-Rink, E.O. Göbel, Optics of Semiconductor Nanostructures, Akademie Verlag, Berlin 1993.

[2] A. Konin, J. Phys., Condens. Matter 19, 016214 (2007).

[3] K. Kash, J.M. Worlock, M.D. Sturge, P. Grabbe, J.P. Harbison, A. Scherer, P.S.D. Lin, Appl. Phys. Lett. 53, 782 (1988).

[4] K. Brunner, G. Abstreiter, M. Walther, G. Böhm, G. Tärnkle, Surf. Sci. 267, 218 (1992).

[5] J.Y. Marzin, J.M. Gerard, A. Izrael, D. Barrier, G. Bastard, Phys. Rev. Lett. 73, 716 (1994).

[6] V.L. Bonch-Bruevich, S.G. Kalashnikov, Physics of Semiconductors, Nauka, Moscow 1977 (in Russian).

[7] G.P. Peka, Physical Effects on Semiconductor Surface, Vissha Shkola, Kiev 1984 (in Russian). 